Karolina JANOWSKA ${ }^{1}$

Uniwersytet Gdański

\title{
Evolución de la metodologia de la enseñanza de las lenguas extranjeras
}

\begin{abstract}
Summary
This article reviews the evolution of the most popular methods of teaching foreign languages in Europe. The history of language teaching was marked by continuous methodological changes resulting from the search for an ideal teaching method and various language currents and psychological approaches prevailing at the time of their occurrence. This discipline went through various stages, the most significant of which took place in the twentieth century, to develop a new method for more effective learning, which led to the development of a methodology that would provide clarification of answers to questions raised in the field of language learning. In recent years, what has been distinguished is a task-based language teaching field that provides students with ideal opportunities for developing communication skills and effective learning.
\end{abstract}

Keywords: foreign language teaching, method, approach, didactic techniques, task-based language teaching, traditional method or grammar-translation method, Cognitive and Constructivist Paradigm

\section{Introducción}

Antes de presentar los métodos y enfoques más populares usados para enseñar las lenguas extranjeras cabe explicar que significa la terminología "método".

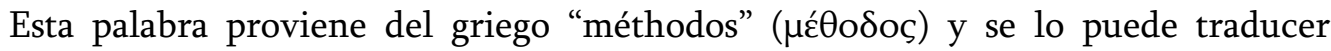
como "camino" o"proceso". Estos dos significados pueden referirse (Alcade Mato 2011: 9):

(...) en el marco de la didáctica de lenguas, tanto a una corriente metodológica de análisis lingüístico, como a un manual o libro de texto, entre otros contenidos existentes, como puede ser la referencia al procedimiento de aprendizaje, diferenciándose, en este sentido, al método inductivo del deductivo.

Podemos decir que en este sentido, el concepto de los métodos de enseñanza tenía muchas interpretaciones y definiciones diversas. Según una de ellas el metodo "en el contexto de procesos de enseñanza/aprendizaje es camino de la enseñanza formado de forma sistemática y metódica" (ibídem). De manera similar se usa la terminología "enfoque" como sinonimo de la palabra "método"

1 (D) https://orcid.org/0000-0002-5539-2318. 
en el sentido de perspectiva metodoógica. Como dijo uno de los autores (Melero Abadía 2000: 15):

Muchas veces se denomina "método" a lo que en realidad es un enfoque que toma como base de reflexión teórica sobre la lengua y/o sobre el aprendizaje, y en él encontramos más espacio para la interpretación y variación individual de lo que permitiría un método. Esta es la razón por la cual hoy denominamos más acertadamente "Enfoque Comunicativo" a la corriente metodológica que en sus inicios se denominó "Método Comunicativo", por ejemplo.

\section{Método tradicional o método gramática-traducción}

Por lo tanto, el término "método" debe entenderse como el proceso o la manera de lograr un objetivo específico, mientras que el "enfoque" consistiría en una perspectiva adoptada como punto de vista para un enfoque de un tema determinado.

Para poder presentar el método tradicional haría que referirse a la historia de la enseñanza de lenguas extranjeras en Europa desde los comienzos de la época moderna. No podemos olvidar que hasta el siglo XVI la enseñanza era reducida al latín y griégo, que formaban la base de la educación. Como en la mayoría de los casos, los maestros de lenguas clasicas en estos tiempos tenían formación teológica y no filológica, la metodología utilizada por ellos en las clases de latín era la qye hoy esta conocida como "método tradicional". En este metodo "el estudiante asistía a la clase prácticamente como oyente, con total carencia de participación, y las clases consistían en la lectura de un texto, con su correspondiente análisis y comentario posterior por parte del maestro" (Alcade Mato 2011: 11).

Este método es también conocido como "gramática - traducción". Como era utilizado sobre todo en el aprendizaje de las lenguas clásicas, de aqui es llamado "el método tradicional". Su objeto principal es en primer lugar conocimiento de las palabras y reglas gramaticales del idioma de destino para que el estudiante pueda comprender las oraciones, construirlas y, por lo tanto, apreciar la cultura y la literatura de una lengua extranjera, al tiempo que adquiere un conocimiento más profundo de los suyos y desarrolla su intelecto y capacidad de raciocinio. Lo caracteristico de este método es que las clases son en el idioma materno con poco uso del idioma extranjero y las palabras se enseña en forma de lista.

Segun los autores (Sánchez, 2009: 45-48, Richards y Rodgers, 2003: 15-16) las reglas principales de este metodo son siguientes: 
- El objetivo de la enseñanza es la literatura, a la cual se accede a partir de la memorización de listas de palabras y reglas gramaticales, que son presentadas de forma deductiva.

- Se prestará, por tanto, poca o ninguna a atención a las destrezas de expresión oral y comprensión auditiva (hablar y escuchar).

- La lengua de enseñanza es la primera lengua del aprendiente y se tendrá especial cuidado con la corrección de las traducciones.

- El profesor es el protagonista y autoridad en la clase y, por tanto, el alumno es solo un agente pasivo. Su papel es el de recibir, asimilar y acumular conocimientos.

- Las actividades de clase giran en un torno a la secuencia explicacióncomprensiónpráctica.

- No se toleran los errores; se corregirán de inmediato, pues la repetición del error llevaría a su asentamiento y consolidación.

Desde un punto de vista teórico, este método consiste en enseñar la segunda lengua a través de la primera, porque toda la información necesaria para construir una oración o comprensión del texto se facilita mediante explicaciones en la lengua materna del estudiante. Segun este modelo se puede diferenciar tres pasos esenciales para el aprendizaje de la lengua (Alcade Mato 2011: 12):

1. la memorización previa de un listado de palabras,

2. el conocimiento de las reglas gramaticales necesarias para unir y relacionar esos vocablos en oraciones correctas,

3. la realización de ejercicios, básicamente de traducción, tanto directa como inversa.

Por lo tanto, solo tratamos con el método deductivo, en el que siempre partimos de la regla para dar un ejemplo. La base es la forma escrita del lenguaje, que esencialmente sigue siendo literaria y, como tal, se guía por criterios formales, y la nota es prácticamente nula con respecto a los aspectos de pronunciación y entonación, excluyendo, igualmente, todas las sugerencias con respecto al aspecto de comunicación.

A finales del siglo XIX los especialistas en la enseñanza de idiomas empezaron a buscar nuevas formas de enseñar las lenguas, aunque este metodo era utilizado hasta bien terminada la Segunda Guerra Mundial. Es todavía usado en algunas partes del mundo porque exigue poco de los profesores, sin embargo puede llegar a crear frustración en los estudiantes (Richards y Rodgers, 2003: 16).

Para comprender por qué este método ha sido el líder entre los métodos de enseñanza durante tanto tiempo, debemos recordar que la enseñanza de lenguas extranjeras hasta mediados del siglo pasado fue un privilegio de la educación superior o las escuelas de "élite" dentro de la educación pública, y por lo tanto el 
hecho de que el método de "gramática y traducción" fue considerado el más adecuado para aprender idiomas de una manera "cultural", fue una garantía de éxito en tales instituciones.

\section{Paradigma cognitivista y constructivista}

Para discutir sobre el paradigma cognitivista y constructivista sería recommendable presentar la terminogía. El constructivismo es un paradigma relacionado con el desarrollo cognitivo, y sus raíces llegan a la teoría de Piaget sobre el desarrollo de la inteligencia, llamada epistemología genética, en la que la génesis del conocimiento es el resultado de un proceso dialéctico de asimilación, adaptación, conflicto y equilibrio. El paradigma constructivista, por lo tanto, asume que el conocimiento es un constructo mental que es el resultado de la actividad cognitiva de un estudiante que ha estudiado. El cognitivsmo, por otro lado, es de gran importancia para el estudio de la enseñanza y el aprendizaje, contribuyendo al conocimiento preciso de ciertas habilidades básicas de aprendizaje, como la atención, la memoria y el razonamiento. Estas teorías fueron precedidas por las teorías donducistas y estructuralistas, pero en la segunda mitad del siglo XX las teorias mencionadas dejaron de tener el efecto que habían anterioramente. Como dijo uno de los autores (Ortiz Parada 2014: 17):

Esto fue debido, principalmente, a dos razones: por parte de la lingüística, a la aparición del generativismo, encabezado por N. Chomsky y, por parte de la psicología, la emergencia del cognitivismo y su interés por el procesamiento mental de la información. Ambas corrientes, además, apostaban por el estudio del significado, al contrario que el estructuralismo, que se centraba más en la forma:

- Se puede decir que los elementos de la teoría cognitiva, que era una novedad, se reflejaron en los estudios de diversas ciencias. Este modelo de enseñanza se caracterizó por las siguientes características (íbidem):

- Una participación activa del alumno.

- Se tienen en cuenta los procesos cognitivos que favorecen el aprendizaje, así como el uso de distintas estrategias para reorganizar los contenidos.

- Se reconoce que cada persona posee unos procedimientos propios para el aprendizaje, por lo que habrá que potenciar el desarrollo de los recursos propios de cada alumno.

- Fomento de la autoestima del aprendiente, pues es necesario que muestre confianza hacia lo que es capaz de hacer y esté preparado para resolver problemas, comunicarse y aprenda a aprender. 
En la metodología y didáctica de los idiomas, esta teoría es la base de un enfoque humanista y de la enseñanza del lenguaje comunicativo, que serán analizados a continuación.

Aqui vamos a presentar el enfoque humanista. Según los autores (Sánchez, 2009: 205-206):

Los enfoques humanísticos son un conjunto de métodos surgidos en torno a los años 70, que, a diferencia de los métodos anteriores -que se basaban en aspectos relacionados con la lengua y su naturaleza-, se centran en otros aspectos nolingüísticos como el aprendizaje, las características de los alumnos, la motivación como factor de aprendizaje, etc.

Cabe entonces presentar los métodos en los que se basa el enfoque humanista. Son "la sugestopedia, el aprendizaje de la lengua en comunidad o comunitario, la respuesta física total y el método silencioso" (Richards y Rodgers 2003:75). Antes de presentarlos de manera más precisa vamos a enumerar sus características que son siguientes (Ortiz Parada 2014: 15):

- Se apoyan principalmente en la Psicología, más que en la Lingüística.

- Se tienen en cuenta los factores afectivos y emocionales de los alumnos.

- Promueven un entorno de aprendizaje en el que se reduzca la ansiedad y aumente la confianza personal.

- Defienden el aprendizaje basado en la experiencia: el estudiante solo aprende lo percibe como relevante y forma parte activa de él. Este aprendizaje será además duradero.

- Consideran que la autoevaluación por parte de los aprendientes fomenta en ellos la independencia, la motivación y la confianza.

A pesar de sus modernos supuestos de novedad en el enfoque, estos métodos no se han encontrado con la recepción con la que contaron sus creadores. Sin embargo, muchos de sus ideales han influido positivamente en varias perspectivas metodológicas.

Uno de los metodos utilizados por enfoque humanista era la sugestopedia. Es conocida también como método sugestopédico. Era introducida por el psiquiatra búlgaro G. Lozanov en 1978. Este método basa en las ideas para "el control mental de las influencias irracionales e inconscientes y reconducirlas para un correcto aprendizaje" (Richards y Rodgers, 2003: 101). Este enfoque está particularmente vinculado al entorno de aprendizaje en el que elementos como los muebles, la decoración, la iluminación o el uso de la música contribuyen a la creación 
de un clima de aprendizaje adecuado. La sugestopedia pone más énfasis en el vocabulario y la traducción que en el contexto lingüístico. Su teoría de aprendizaje es basada en un proceso de "desugestión" y posterior "suggestion" (Ortiz Parada 2014: 16). Estos dos procedimientos pueden accera la memoria a través de los elementos siguientes (Rodgers, 2003: 102-103):

- Autoridad de la institución educativa y del profesor. El uso de tecnicismos y de datos empíricos por parte de los profesores, convencidos de las bondades del método, despierta el interés de los aprendices y los predispone a una mayor receptividad.

- Proceso de "infantilización". Un método de estas características despierta en los estudiantes la confianza en sí mismos y la espontaneidad para participar en simulaciones, juegos y canciones.

- Doble plano. El entorno en el que tiene lugar la enseñanza adquiere tanta importancia como los propios contenidos de aprendizaje.

- Entonación y ritmo. Variar ambos contribuye a crear en los alumnos un estado de "pseudopasividad de concierto" en el que se dan las condiciones óptimas para el aprendizaje, gracias a la liberación de la ansiedad y la tensión por parte de ellos.

En este método el papel de los profesores es mediar y dirigir el aprendizaje para crear un clima de confianza en sí mismo y relajación en el aula creando situaciones que lo favorezcan. Las principales quejas que este método ha recibido surgían de la falta de exactitud científica de sus teorías y procedimientos operativos.

Otro metodo utilizado en el enfoque humanista es el aprendizaje comunitario o aprendizaje de la lengua en comunidad. Era creado por psicólogo C.A. Curran en 1976. Su nombre proviene del concepto de aprendizaje entendido como un proceso en el que la cooperación de los miembros del grupo contribuye a su logro. En este concepto, el profesor y los estudiantes forman una comunidad en la que cooperan para lograr sus objetivos (Ortiz Parada 2014: 17).

Sobre este método los autores dijeron de manera siguiente (Richards y Rodgers, 2003: 94):

Curran no especificó ninguna teoría sobre la lengua en la que basarse, pero sí lo intentó su discípulo La Forge, al definir la comunicación como: [algo] "más que la simple transmisión de un mensaje desde el emisor al receptor [...] supone no solamente la transferencia unidireccional de información a otra persona, sino la propia relación entre los hablantes"

El creador de este método se refirió principalmente al aspecto interactivo de los idiomas, realizando una investigación sobre las interacciones que ocurren entre los estudiantes y las que ocurren entre ellos y los profesores. 
Entre los métodos de eseñanza las lenguas extranjeras del enfoque humanista cabe mencionar la respuesta física total, elaborada en 1977 por J. Asher, profesor de psicología de la Universidad Estatal de San José en California. Este autor se basó en varias propuestas previas de aprendizaje y agregó que con la actividad física es posible aprender un nuevo idioma. La teoría de la enseñanza de Asher es basada en tres hipotesis en las que el autor definó los elementos que lo pueden facilitar o dificultar. Ellas son siguientes (Richards y Rodgers, 2003: 78-79):

- Poseemos un bioprograma innato que define la forma óptima en la que se adquirirán la primera y la segunda lengua. Así, al igual que los niños comprenden antes que hablan gracias a los movimientos físicos que deben realizar para cumplir las órdenes que reciben de sus padres, los adultos realizarán ejercicios de compresión oral combinados con actividades dinámicas. Las habilidades productivas vendrán después.

- Existe una lateralización de los hemisferios del cerebro que hace que tengan funciones distintas. De esta forma, paralelamente a lo que decíamos antes, una vez que se haya desarrollado lo suficiente la comprensión lingüística a través de actividades físicas y de lo cual se encarga el hemisferio derecho, el hemisferio izquierdo se activará para dar lugar a la producción lingüística.

- Un aprendizaje pleno tendrá lugar de mejor forma en un contexto libre de estrés. La adquisición de la primera lengua se da en un ambiente libre de presiones, mientras que en el aprendizaje de una segunda lengua se dan situaciones de tensión y ansiedad, por lo que habrá que promover actividades relajantes y agradables para los aprendientes.

El propósito principal de este método es enfocarse en el desarrollo de la competencia oral de los estudiantes. Sin embargo, en relación con el contenido del lenguaje, se basa en elementos léxicos y gramaticales. Sin embargo, a diferencia del enfoque estructuralista, este método pone mucho más énfasis en el contenido y el significado que en la forma (íbidem). En cuanto a los roles de maestros y estudiantes, el papel principal de estos últimos fue escuchar las instrucciones dadas por el profesor e implementarlas a práctica. Por otro lado, el papel del profesor es activo y directo, porque es él que decide lo que el estudiante está aprendiendo y, por lo tanto, el profesor presenta y organiza los materiales. Según la teoría de Asher (Ortiz Parada 2014: 19):

El profesor será el encargado de dar las oportunidades de aprendizaje, más que de enseñar directamente De forma similar a como aprende un niño con sus padres, al principio corregirá muy poco los errores de los estudiantes, puesto que esto 
les inhibe, para ir controlando más sus producciones según vayan progresando sus capacidades.

La respuesta física total ha sido un tanto exitosa entre los defensores del papel de entender idiomas extranjeros en la ciencia. A pesar de esto, se ha cuestionado la validez de las afirmaciones que este método sugiere practicar en el aula en relación con las necesidades reales de los estudiantes.

Ultimo método utilizado en el enfoque humanista es el método silencioso, también conocido como método del silencio. Era propuesto en 1972 por el profesor C. Gattengo. Según la idea principal de este método el profesor "debe guardar silencio, interfiriendo lo menos posible en las actuaciones de los alumnos y animándole a producir tantos enunciados como sea possible" (Sánchez, 2009: 222).

Como los métodos anteriores este también es basado en diversas teorías del aprendizaje. Los autores enumeran tres métodos (Richards y Rodgers, 2003: 85-86):

- De Bruner toma la teoría del "aprendizaje por descubrimiento", que defiende que el aprendizaje tiene lugar de mejor forma si el alumno descubre o crea lo que tiene que aprender, en lugar de recordarlo o repetirlo.

- Retoma las regletas y cuadros de colores de Cuisenaire para la enseñanza de la pronunciación, pues estos recursos visuales crean asociaciones mentales que facilitan su memorización.

- Niega el enfoque natural del aprendizaje de lenguas y propone un "enfoque artificial". Según este, el proceso de aprendizaje de una segunda lengua es totalmente distinto al de la primera, pues requiere un compromiso personal por parte del alumno en su desarrollo.

Por otro lado, el profesor adoptó un punto de vista estructuralista del lenguaje, que se percibe como un conjunto de sonidos que, gracias a reglas gramaticales específicas, crean la orden de oración. En este enfoque el profesor era mediator del aprendizaje mientras el papel del alumno es activo: es él que tiene responsibilidad por su progreso y tiene que controlar su producción.

\section{Paradigma conductista}

Entre los metodos de la ensenanza de las lenguas mas populares desde final del siglo XIX hasta la mitad del siglo XX era sin duda el paradigm conductista. Su creador fue psicólogo americano B.Skinner en 1975. Este paradigma era basado en la teoría del conductismo. El supuesto principal de esta teoría es que todo el proceso del aprendizaje es nada más que el efecto de la imitación y repetición 
una serie de respuestas gracias a los estímulos concretos. El succeso o fracaso de todo el proceso dependen del grado de acceptacion de las respuestas (Ortiz Parada 2014:7). Esta teoría es por lo tanto una base para los enfoques estructuralistas y también para el método natural.

No podemos olvidar que el paradigma condictista era una parte del movimiento de reforma en la enseñanza de las lenguas extranjeras. Como tal se resumía en los postulados siguientes (ibídem):

- preeminencia de la lengua hablada y escuchada sobre la escrita,

- aplicación de las aportaciones de la fonética a la enseñanza y a la formación de profesores,

- presentación de las palabras en oraciones y en contextos significativos,

- enseñanza inductiva de la gramática y rechazo a la traducción.

En la base del paradigm concuctista surgieron los novedosos metodos de ensenanza entre los que podemos sustituir sobre todo el método natural y los enfoques estructuralistas.

El método natural apacerio en 1860 cuando el lingüista francés L. Sauveur fundó una escuela de enseñanza. Fue él que descubrió similitud existente entre el proceso de aprendizaje de la lengua materna y una lengua extranjera y por lo tanto empezó a emplear este método. Según su teoria "no es necesario recurrir a la L1 para aprender otra lengua, pues el significado puede ser transmitido a través de su presentación y ejemplificación en la LE” (íbidem). Podemos decir entonces que el método natural adicionalmente se basa en la repetición y hasta que los alumnos tengan un conocimiento suficiente del idioma oral, no puede pasar al lenguaje escrito ni a la gramática. Los representantes más conocidos de este método son el método directo y el método Berlitz.

Las raíces del método directo se remontan al principio del siglo XX cuando diferentes académicos de Francia, Alemania y Estados Unidos lo han desarrollado. Este metodo se caracteriza por siguientes rasgos (Sánchez, 2009: 58-61):

- Uso exclusivo de la lengua meta, por lo que la traducción queda totalmente excluida.

- Preferencia de la lengua oral sobre la escrita.

- Enseñanza del vocabulario y las estructuras cotidianas y siempre en entornos naturales, en un contexto en el que adquieren sentido pleno.

- La gramática se enseña de modo inductivo.

- Énfasis en la pronunciación y la gramática. 
- Los errores de corrigen de inmediato, pero de una forma menos brusca que en el método anterior, pues la corrección no se concibe como un castigo, sino como una mejora en la eficacia comunicativa.

Por lo tanto, podemos decir que el método directo de contraste con el método de gramática - traducción es dinámico y promueve la interacción. De acuerdo con este método la enseñanza está abierta; lo significa que los profesores no imparten lecciones de forma autoritaria. Los alumnus participant en las clases de forma activa y responden a las preguntas de los profesores y de sus compañeros. Sin embargo es el profesor que organiza las clases y no los alumnos.

Segun algunos autores las principales críticas de este metodo "se centran en su falta de base teórica y metodológica, pues no se asienta en ninguna teoría sobre la naturaleza de la lengua y su aprendizaje ni posee fundamentos metodológicos que sirvan de base a las técnicas de enseñanza de las que se vale" (Richards y Rodgers, 2003: 22).

El segundo método natural, el metodo Berlitz, apareció en 1878 cuando en especialista de las lenguas extranjeras, M. Berlitz, fundó en Rhode Island la primera escuela basada en este método. Al llegar a EE.UU Berlitz descubrió que un profesor de francés inseñaba a este lengua sin utilizar inglés. Por lo tanto el profesor aleman aprovechó este método lo aplicó a otras muchas lenguas.

Los rasgos de este método son siguientes (Sánchez, 2009: 56):

- Predominio de la lengua oral sobre la escrita.

- Negación de la traducción.

- La gramática no se enseña hasta poseer un cierto dominio de la lengua.

- Empleo de la conversación y la técnica pregunta-respuesta.

- Los profesores deben ser nativos del idioma que enseñan.

Respecto al método promovido por L. Sauveur, Berlitz le presentó algunas novedades, además de organizar los materiales de una manera más racional y ordenada. Por lo tanto, se puede decir que este método era más accesible para los maestros con menos preparación de un campo determinado.

El método natural también incluye los enfoques estructuralistas. Ellos se basan en las teorías lingüísticas del estructuralismo de corte conductista (Ortiz Parada 2014:10), es decir en los metodos de enseñanza en los que las lenguas son consideradas como unas estructuras que se puede aprender a través de repetición y conductas. Los métodos utilizados en estos enfoques son el audiooral, la enseñanza situacional de la lengua en Inglaterra y el método SGAV o global-estructural. 
El método audio-oral surgió como una de las consecuencias de segunda Guerra Mundial porque los americanos tuvieron la necesidad de aprender lenguas de una forma rápida y eficaz. Por lo tanto había que crear un nuevo método de aprendizaje las lenguas extranjeras, pues en este momento no existía un metodo que cumpliría las condiciones preestablecidas. Los primeros intentos de desarrollar este tipo de métodos tuvieron lugar en 1933 cuando el lingüista Bloomfield creó teorías sobre el aprendizaje de las nuevas lenguas que podian influir en el desarrollo del Programa para la Formación Especializada del Ejército (Richards y Rodgers, 2003: 58).

Debido a que este programa no cumplió con las necesidades militares, los especialistas de la enseñanza de lenguas extranjeras dijeron que se deberían hacer algunos cambios en él para que tuviera efecto en un ámbito militar. En 1945 el lingüista C.C. Fries, colaborando con otros investigadores de la Universidad de Michigan creó el método audio-oral. Este método se basaba en el análisis contrastivo de las estructuras de las lenguas y su objetivo principal era "la enseñanza de la lengua a partir de la repetición sistemática de las estructuras básicas de las oraciones, prestando especial atención a la pronunciación" (Ortiz Parada 2014: 11).

En los años 60 del siglo XX finalmente nació otro metodo de la ensenanza de las lenguas extranjeras - el método audiolingüístico o audiolingüe. Las razones principales para crear este método incluyen la necesidad de aprender rápidamente un idioma extranjero y también la expansión del inglés por el mundo hacía que la sociedad tuviera que aprenderlo. Este nuevo método partía de las teorías estructuralistas y de análisis contrastivo de las lenguas que promovía el método audio-oral. Se caracterizaba por los rasgos siguientes (Richards y Rodgers, 2003: 69-71):

- Prevalece la lengua oral sobre la escrita.

- El profesor es el protagonista de la clase, controla el contenido, el ritmo y el estilo del aprendizaje.

- La gramática no se enseña, sino que se adquiere de modo inductivo, es decir, debe surgir de la práctica.

- Rechazo de la lengua materna y, por tanto, de la traducción para evitar interferencias.

- El contenido de la clase está fijado de antemano, pero es aconsejable alternar diversos tipos de actividades para evitar la desmotivación en los alumnos.

- El vocabulario y las estructuras que se aprenden son los más frecuentes y debe hacerse siempre dentro de un contexto.

- Los errores se corrigen de inmediato, pues su repetición haría que se consolidasen como tales. 
Podemos decir que este método era más popular en EE.UU. en años 60 mientras en Europa todo el tiempo los expertos seguian utilizando el metodo natural y el metodo directo. Más tarde, los investigadores concluyeron que este nuevo método no era una solución esperada en la enseñanza de idiomas. Como dijo uno de los autores (Ortiz Parada 2014: 12):

La segunda crítica importante hecha a este método se basa en la falta de resultados satisfactorios que los que usaban este método esperaban. Veían que muchos de sus alumnos no eran capaces de utilizar la lengua que habían aprendido de forma autónoma fuera del aula y muchos estudiantes consideraban, también, esta forma de enseñanza aburrida e ineficaz.

Por lo tanto, fue necesario seguir buscando un método más efectivo. Entre los años 20 y 30 en Gran Bretaña surgió en método llamado enfoque oral que basaba en tres principios sobre los contenidos del curso: la selección, gradación y presentación. A partir de los años 50 este enfoque evolucionó hacia la enseñanza situacional de la lengua.Los rasgos principales de este método son siguientes (Richards y Rodgers, 2003: 50-55):

- Como en los anteriores, predominio de la lengua oral sobre la escrita.

- Se desaconsejan las explicaciones, pues se espera que el alumno deduzca las estructuras y los significados a partir de la situación en la que esté inserta.

- Los errores se corrigen de inmediato para no consolidar malos hábitos, como es natural en los métodos de corte conductista.

- El profesor es el protagonista de la clase y en una fase más avanzada del curso los alumnos adquieren un papel un poco más activo al poder controlar las preguntas y respuestas con sus compañeros.

- La clase se basa en el modelo P-P-P: presentación de la unidad objeto de aprendizaje en su contexto, práctica de esa unidad y producción de otras unidades de forma más libre.

Según este enfoque la lengua es una actividad el lenguaje es una actividad relacionada con metas y situaciones de la vida real, y todo el contenido de enseñanza en el aula debe aparecer en contexto para que los alumnos puedan usar el lenguaje en situaciones reales fuera del aula.

A partir de mediados de los años 60 se comenzó a alejarse de la enseñanza situacional, en favor de la enseñanza comunicativa de la lengua. Ultimo de los métodos presentados como parte de los enfoques estructuralistas era método estructuro-global-audiovisual llamado también SGAV, del francés StructuroGlobale Audio-Visuelle (Ortiz Parada 2014: 12). Este metodo apareció en Francia 
en los años 50 como la conexión del método audiovisual y el verbo - tonal. Por otro lado este método tenia también algunas caracteristicas del método directo. Los rasgos principals de este metodo son siguientes (Sánchez, 2009: 88-91):

- El objetivo es el dominio de ciertas estructuras gramaticales, base para la comunicación en una lengua extranjera.

- Los sentidos (oído y vista) son el filtro por el cual aprehendemos las lenguas.

- Uso de materiales audiovisuales (dibujos, películas, diapositivas, etc.) para evitar el uso de la lengua materna y asociar directamente las palabras con su referente.

- El aprendizaje se hace de forma global, procesando primero los elementos aislados y después se unen en un todo superior (global).

- El profesor es el modelo que los alumnos deben conseguir reproducir a través de la imitación, y la repetición.

Se puede destacar dos contribuciones de este sistema de enseñanza: el profesor utiliza un libro, que le sirve de guía para la preparación de las clases y se instalan los laboratorios de idiomas para la práctica de las lenguas. El método SGAV sin embargo perdió fuerza ante las críticas entre unos años de su esplendor, sobre todo por sus teorías de aprendizaje que basaban en la repetición y la visión estereotipada que aportan sus diálogos.

\section{Referencias bibliográficas}

Alcalde Mato, N. (2011). Principales metodos en ensenanza de lenguas extranjeras en Alemania. Revista de Lingüística y Lenguas Aplicadas. (vol. 6). https://dialnet.unirioja.es/descarga/articulo/4779301.pdf [acceso: 19.06.2018].

Melero Abadía, P. (2000). Métodos y enfoques en la enseñanza/aprendizaje del español como lengua extranjera. Madrid.

Ortiz Parada, M. (2014). Nuevas tendencias metodológicas en la enseñanza de lenguas extranjeras. Trabajo de fin de grado. Universidad de Cádiz.

Richards J., Rodgers T.S. (2003). Enfoques y métodos en la enseñanza de idiomas. Madrid.

Sánchez Pérez, A. (1992). Historia de la enseñanza del español como lengua extranjera. Madrid. 
